\title{
Article \\ Immune Cells Invade the Collateral Circulation during Human Stroke: Prospective Replication and Extension
}

\author{
Marc Strinitz ${ }^{1}$, Mirko Pham ${ }^{1}$, Alexander G. März ${ }^{1}$, Jörn Feick ${ }^{1}$, Franziska Weidner ${ }^{1}$, Marius L. Vogt ${ }^{1}$, \\ Fabian Essig ${ }^{2}$, Hermann Neugebauer ${ }^{2}$, Guido Stoll ${ }^{2}$, Michael K. Schuhmann ${ }^{2}$ \\ and Alexander M. Kollikowski ${ }^{1, * \mathbb{D}}$ \\ 1 Department of Neuroradiology, University Hospital of Würzburg, 97080 Würzburg, Germany; \\ Strinitz_M@ukw.de (M.S.); Pham_M@ukw.de (M.P.); Maerz_A@ukw.de (A.G.M.); Feick_J@ukw.de (J.F.); \\ Weidner_F1@ukw.de (F.W.); Vogt_M2@ukw.de (M.L.V.) \\ 2 Department of Neurology, University Hospital of Würzburg, 97080 Würzburg, Germany; \\ Essig_F@ukw.de (F.E.); Neugebauer_H@ukw.de (H.N.); Stoll_G@ukw.de (G.S.); \\ Schuhmann_M@ukw.de (M.K.S.) \\ * Correspondence: Kollikowsk_A@ukw.de
}

Citation: Strinitz, M.; Pham, M.; März, A.G.; Feick, J.; Weidner, F.; Vogt, M.L.; Essig, F.; Neugebauer, H.; Stoll, G.; Schuhmann, M.K.; et al. Immune Cells Invade the Collateral Circulation during Human Stroke: Prospective Replication and Extension. Int. J. Mol. Sci. 2021, 22, 9161. https://doi.org/10.3390/ijms 22179161

Academic Editor: Antonino Tuttolomondo

Received: 28 July 2021

Accepted: 24 August 2021

Published: 25 August 2021

Publisher's Note: MDPI stays neutral with regard to jurisdictional claims in published maps and institutional affiliations.

Copyright: (c) 2021 by the authors. Licensee MDPI, Basel, Switzerland. This article is an open access article distributed under the terms and conditions of the Creative Commons Attribution (CC BY) license (https:// creativecommons.org/licenses/by/ $4.0 /$ )

\begin{abstract}
It remains unclear if principal components of the local cerebral stroke immune response can be reliably and reproducibly observed in patients with acute large-vessel-occlusion (LVO) stroke. We prospectively studied a large independent cohort of $n=318$ consecutive LVO stroke patients undergoing mechanical thrombectomy during which cerebral blood samples from within the occluded anterior circulation and systemic control samples from the ipsilateral cervical internal carotid artery were obtained. An extensive protocol was applied to homogenize the patient cohort and to standardize the procedural steps of endovascular sample collection, sample processing, and laboratory analyses. $\mathrm{N}=58$ patients met all inclusion criteria. (1) Mean total leukocyte counts were significantly higher within the occluded ischemic cerebral vasculature (I) vs. intraindividual systemic controls (S): $+9.6 \%, \mathrm{I}: 8114 / \mu \mathrm{L} \pm 529$ vs. S: $7406 / \mu \mathrm{L} \pm 468, p=0.0125$. (2) This increase was driven by neutrophils: $+12.1 \%$, I: $7197 / \mu \mathrm{L} \pm 510$ vs. S: $6420 / \mu \mathrm{L} \pm 438, p=0.0022$. Leukocyte influx was associated with (3) reduced retrograde collateral flow $\left(\mathrm{R}^{2}=0.09696, p=0.0373\right)$ and (4) greater infarct extent $\left(R^{2}=0.08382, p=0.032\right)$. Despite $L V O$, leukocytes invade the occluded territory via retrograde collateral pathways early during ischemia, likely compromising cerebral hemodynamics and tissue integrity. This inflammatory response can be reliably observed in human stroke by harvesting immune cells from the occluded cerebral vascular compartment.
\end{abstract}

Keywords: ischemic stroke; cerebral ischemia; mechanical thrombectomy; large vessel occlusion; leukocytes; neutrophils; collateral circulation

\section{Introduction}

The cumulative incidence of cerebrovascular diseases is considerable in high-income countries (218 (95\% CI 214-221) per 100,000 in men and 127 (95\% CI 125-128) per 100,000 in women, respectively) and has reached epidemic levels in low to middle-income countries [1,2]. Among these diseases, acute ischemic stroke (AIS) is the leading cause of death and disability, which, despite recent major advances in acute stroke therapy, prompts the urgent need for adjunct treatments beyond macrovascular recanalization [3,4]. In AIS, the immune system exerts a strong and early inflammatory response, which is directed at the ischemic brain [5,6]. Immune cell infiltration into the ischemic brain represents a central component of this response [7], which has been widely and repeatedly observed in the most commonly practiced experimental models of ischemic stroke across species and over decades of stroke research [8,9]. However, detailed spatiotemporal information on immune cell infiltration, including its route of traffic, is particularly lacking for the early phase after vascular occlusion and for the human system in general $[5,10]$. There is ample 
evidence from murine intraluminal filament occlusion models closely mimicking human large-vessel-occlusion (LVO) stroke that the modulation of stroke inflammation may be such a promising adjunct therapeutic target [5,11]. Importantly, in experimental settings, the (post) stroke inflammatory response seems to emerge from the cerebral vascular compartment [5]. In acute human stroke, thus far, only sites located remotely from ischemia have been accessible to observation, e.g., by retrieving samples of peripheral blood or of lumbar cerebrospinal fluid (CSF) [12]. Consequently, the inability of human cerebral observation of stroke-induced inflammation in close temporal and anatomical proximity to the ischemic event remains an obstacle for translation [13-16]. Only recently, the ischemic cerebral vasculature of acute stroke patients has become accessible for investigation during mechanical thrombectomy (MT), while, importantly, recanalization still has not taken place: We and few other groups established the method of blood aspiration from within the ischemic cerebral vasculature during MT, which is performed with a distally placed microcatheter at the end of the occlusion phase immediately before therapeutic recanalization is achieved more proximally through stent-embolus-retrieval [17-19]. In principle, this method can expose local pathophysiology for scientific observation if patient-related, interventional, and laboratory confounders are controlled by protocol $[17,20,21]$. However, the reproducibility and consistency of observations made by this approach remain unclear. A recent study used flow cytometry to define relative immune cell populations within the occluded cerebral vasculature of AIS patients but did not report on absolute immune cell numbers [14]. Consequently, our human observation of local neutrophil infiltration could not be reproduced thus far [17]. The present study aims to (1) prospectively assess local immune cell infiltration quantitatively in a large second independent cohort of LVO stroke patients by applying an extensive protocol, which homogenizes the study population and standardizes the procedural steps for quality control. Furthermore, (2) the impact of local immune cell infiltration on hemodynamic-functional (collateral flow) and radiological-structural (infarct extent) parameters is investigated.

\section{Results}

\subsection{Patient Characteristics}

$\mathrm{N}=116$ samples of $n=58$ consecutive LVO stroke patients meeting all pre-specified protocol criteria (58 local cerebral-ischemic (I) vs. 58 systemic control samples (S)) entered data analyses. Demographic, clinical, radiological, therapy- and sampling-related patient characteristics are shown in Table 1. Briefly, the mean patient age was $74 \pm 11$ years. Women were slightly overrepresented (66\%). Hypertension was the most prevalent comorbid disease $(90 \%)$, followed by atrial fibrillation $(60 \%)$. Median clinical stroke severity as measured by the National Institutes of Health Stroke Scale (NIHSS) at hospital admission was 15 (10-19). Median baseline infarct extent as assessed by the pre-interventional Alberta Stroke Program Early CT score (ASPECTS) was 8 (7-9). N = 24 patients (41\%) received intravenous thrombolysis before endovascular therapy for LVO. Median time from symptom onset to groin puncture was 255 (190-325) min. Initial cerebral angiograms revealed $n=38$ $(66 \%)$ middle cerebral artery (MCA) M1 occlusions, $n=17(29 \%)$ proximal M2 occlusions, and $n=10(17 \%)$ internal carotid artery (ICA-T) occlusions, respectively. Median time from symptom onset to local cerebral-ischemic sampling was 295 (231-295) min. The mean time delay of retrograde collateral blood flow (relative time to peak opacification, rTTP) to reach the target site of cerebral-ischemic blood sampling was $3.8 \pm 1.4 \mathrm{~s}$. Final cerebral angiograms revealed technically successful recanalization (mTICI $\geq 2 \mathrm{~b}$ ) in $n=43$ patients $(83 \%)$ after a median onset-to-final-recanalization time of 335 (253-381) min. Mean duration of antegrade blood flow to reach the mid-insular MCA target site after recanalization was $1.3 \pm 0.6 \mathrm{~s}$. Median follow-up ASPECTS after recanalization therapy was 7 (6-8). Median clinical stroke severity as assessed by NIHSS at $72 \mathrm{~h}$ after MT was 13 (4-18). Parenchymatous hematoma ( $>30 \%$ of infarcted tissue) following MT occurred in $n=2$ patients $(3 \%) . \mathrm{N}=4(7 \%)$ patients succumbed during the hospital stay. Detailed results of baseline characteristics are displayed in Table 1. 
Table 1. Characteristics of included patients.

\begin{tabular}{|c|c|}
\hline Demographics & \\
\hline Age, years (SD) & $74(11)$ \\
\hline Male sex, $n(\%)$ & $20(34)$ \\
\hline \multicolumn{2}{|l|}{ Medical history } \\
\hline Hypertension, $n(\%)$ & $52(90)$ \\
\hline Diabetes mellitus, $n(\%)$ & $14(24)$ \\
\hline Hyperlipidemia, $n(\%)$ & $21(36)$ \\
\hline Atrial fibrillation, $n(\%)$ & $35(60)$ \\
\hline Current smoker, $n(\%)$ & $9(16)$ \\
\hline \multicolumn{2}{|l|}{ Baseline medication } \\
\hline Antithrombotic medication, $n(\%)$ & $30(52)$ \\
\hline Antihypertensive drugs, $n(\%)$ & $52(90)$ \\
\hline \multicolumn{2}{|l|}{ Clinical presentation } \\
\hline Systolic blood pressure, $\mathrm{mmHg}$ (IQR) & $158(140-178)$ \\
\hline Diastolic blood pressure, mmHg (IQR) & $80(67-97)$ \\
\hline Heart rate, $\min ^{-1}(\mathrm{SD})$ & $86(23)$ \\
\hline NIHSS at presentation (IQR) & $15(10-18)$ \\
\hline Unknown time of symptom onset, $n(\%)$ & $19(33)$ \\
\hline ASPECTS at presentation (IQR) & $8(7-9)$ \\
\hline \multicolumn{2}{|l|}{ Treatment } \\
\hline IV rt-PA, $n(\%)$ & $24(41)$ \\
\hline Intervention & \\
\hline Onset-to-puncture, $\min (\mathrm{IQR})$ & $255(190-325)$ \\
\hline \multicolumn{2}{|l|}{ Angiographic occlusion location * } \\
\hline $\mathrm{M} 1, n(\%)$ & $38(66)$ \\
\hline $\mathrm{M} 2, n(\%)$ & $17(29)$ \\
\hline ICA, $n(\%)$ & $10(17)$ \\
\hline Tandem occlusion, $n(\%)$ & $5(9)$ \\
\hline rTTp (occlusive condition), s (SD) & $3.2(1.4)$ \\
\hline Successful recanalization, $n(\%)$ & $48(83)$ \\
\hline Onset-to-final-recanalization, $\min (\mathrm{IQR})$ & $335(253-381)$ \\
\hline rTTp (after recanalization), s (SD) & $1.3(0.6)$ \\
\hline \multicolumn{2}{|l|}{ Sampling } \\
\hline Onset-to-distal sampling, min (IQR) & $295(231-295)$ \\
\hline Onset-to-carotid sampling, min (IQR) & $352(275-414)$ \\
\hline \multicolumn{2}{|l|}{ Outcome } \\
\hline ASPECTS post-intervention (IQR) & $7(6-8)$ \\
\hline NIHSS $72 \mathrm{~h}$ post intervention (IQR) & $13(4-18)$ \\
\hline Parenchymatous hematoma, $n(\%)$ & $2(3)$ \\
\hline In-house mortality, $n(\%)$ & $4(7)$ \\
\hline Neurological cause of death, $n(\%)$ & $3(5)$ \\
\hline Non-neurological cause of death, $n(\%)$ & $1(2)$ \\
\hline
\end{tabular}

Categorical data are presented as number $(n)$ with percentage (\%). Continuous data are presented as mean with standard deviation (SD) or median with interquartile range (IQR). Non-standard abbreviations: ASPECTS, Alberta Stroke Program Early CT score; ICA, internal carotid artery; IV rt-PA, intravenous recombinant tissue plasminogen activator; $\mathrm{M} 1 / \mathrm{M} 2$, middle cerebral artery section; min, minutes; $\mathrm{mmHg}$, millimeters of mercury; NIHSS, National Institutes of Health Stroke Scale; rTTP, relative time to peak opacification; s, seconds. * Including multiple sites per patient.

\subsection{Cerebral Immune Cell Recruitment}

The total number of leukocytes in the local cerebral-ischemic (I) samples obtained under occlusive conditions was significantly increased compared with the systemic (S) control samples obtained from the cervical ICA (+9.6\%, I: 8114/ $\mu \mathrm{L}, 95 \%$ CI 7056 to 9173 vs. S: $7406 / \mu \mathrm{L}, 95 \%$ CI 6469 to $8344, p=0.0125)$. This local increase in leukocyte counts within the occluded vascular compartment was primarily driven by a significant increase in the neutrophil subpopulation $(+12.1 \%$, I: $7197 / \mu \mathrm{L}, 95 \%$ CI 6175 to 8220 vs. S: $6420 / \mu \mathrm{L}$, 
95\% CI 5541 to $7298, p=0.0022)$. There was neither a significant difference in the local total number of leukocytes $(-0.4 \%$ men, I: $8091 / \mu \mathrm{L}, 95 \%$ CI 6272 to 9911 vs. women: $8126 / \mu \mathrm{L}, 95 \%$ CI 6763 to $9490, p=0.9751)$ nor a significant difference in the local neutrophil ( $-4.3 \%$, men, I: $6994 / \mu \mathrm{L}, 95 \%$ CI 5303 to 8684 vs. women: $7308 / \mu \mathrm{L}, 95 \%$ CI 5963 to $8653, p=0.7716)$, lymphocyte $(+12.5 \%$, men, I: $694 / \mu \mathrm{L}, 95 \%$ CI 502 to 886 vs. women: $617 / \mu \mathrm{L}, 95 \%$ CI 464 to $769, p=0.3426)$ and monocyte $(-4.4 \%$, men, I: $130 / \mu \mathrm{L}, 95 \% \mathrm{CI}$ 85 to 175 vs. women: $136 / \mu \mathrm{L}, 95 \%$ CI 80 to $193, p=0.5197)$ cell count between men and women. Likewise, there were no significant differences between the control sample and local cerebral-ischemic lymphocyte ( $-8.3 \%$, I: $644 / \mu \mathrm{L}, 95 \%$ CI 527 to 760 vs. S: $702 / \mu \mathrm{L}$, $95 \%$ CI 548 to $856, p=0.8127$ ) or monocyte counts ( $-8.8 \%$, I: $134 / \mu \mathrm{L}, 95 \%$ CI 95 to 173 vs. S: $147 / \mu \mathrm{L}, 95 \%$ CI 96 to 198, $p=0.6341$ ). Total and differential leukocyte counts are shown for each sampling location in Figure 1.
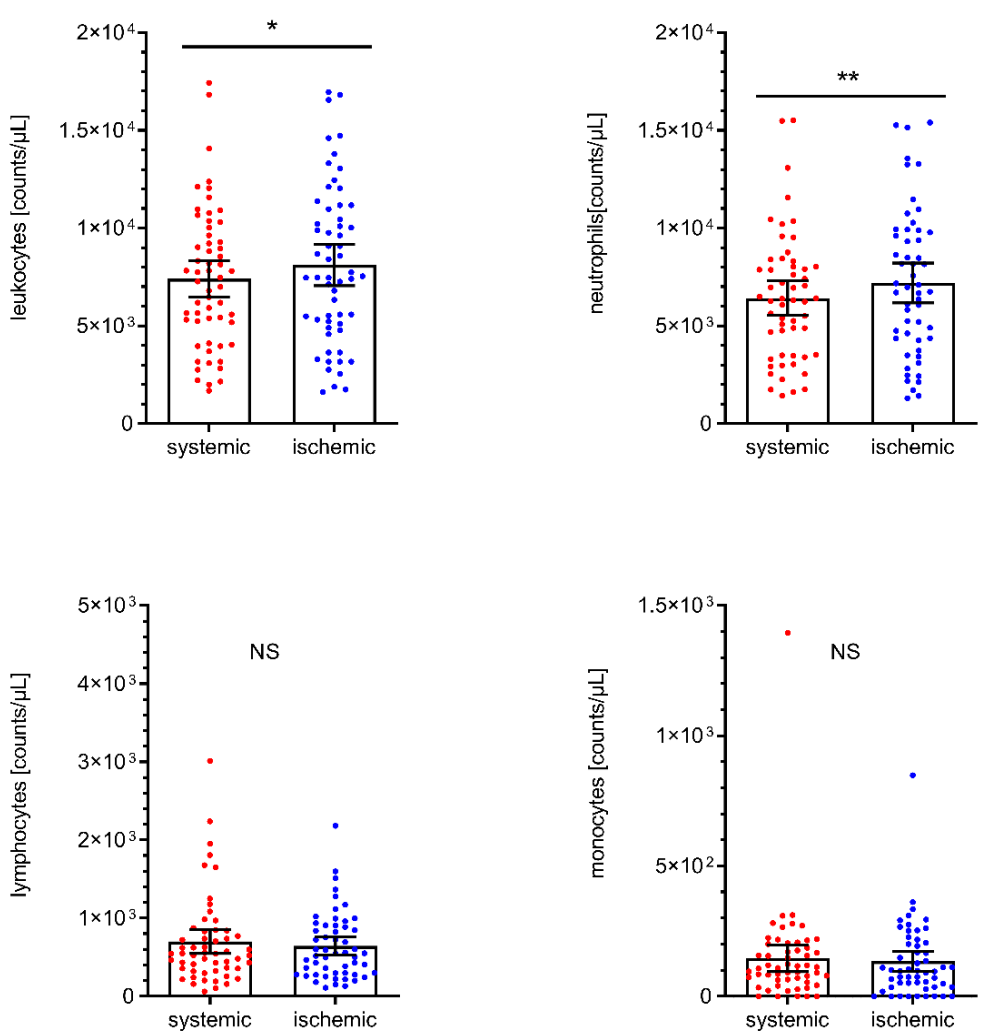

Figure 1. Total leukocyte, neutrophil, lymphocyte, and monocyte cell counts by sampling location. Scatter dot plot with mean and 95\% confidence interval (CI). Total leukocyte cell counts (upper left panel) were tested by paired T-test. ${ }^{* *} p<0.005, n=58$. Differential leukocyte cell counts were tested by the Wilcoxon rank-sum test. ${ }^{*} p<0.05$. NS, not significant.

\subsection{Association of Cerebral Immune Cell Recruitment with Local Blood Flow}

Linear regression was performed to investigate the impact of the observed immune cell influx on retrograde collateral flow under occlusive conditions and on antegrade blood flow following recanalization (rTTP). Local cerebral-ischemic total leukocyte $(\beta=0.0001027$, $\left.R^{2}=0.09696, p=0.0373\right)$ and lymphocyte counts $\left(\beta=0.001095, R^{2}=0.1082, p=0.0313\right)$ were significantly correlated with the collateral transit time to reach the sampling site located distally to the occlusive target lesion, translating into an increase of collateral transit time of $0.5 \mathrm{~s}$ for every increase of 4869 total leukocyte counts $/ \mu \mathrm{L}$. Local cerebral-ischemic neutrophil and monocyte counts were also positively associated with rTTP, however, without statistical significance. None of the local cell counts were correlated with antegrade blood flow measured after the restoration of antegrade flow immediately after recanalization. The results of these analyses are given in Figure 2. 

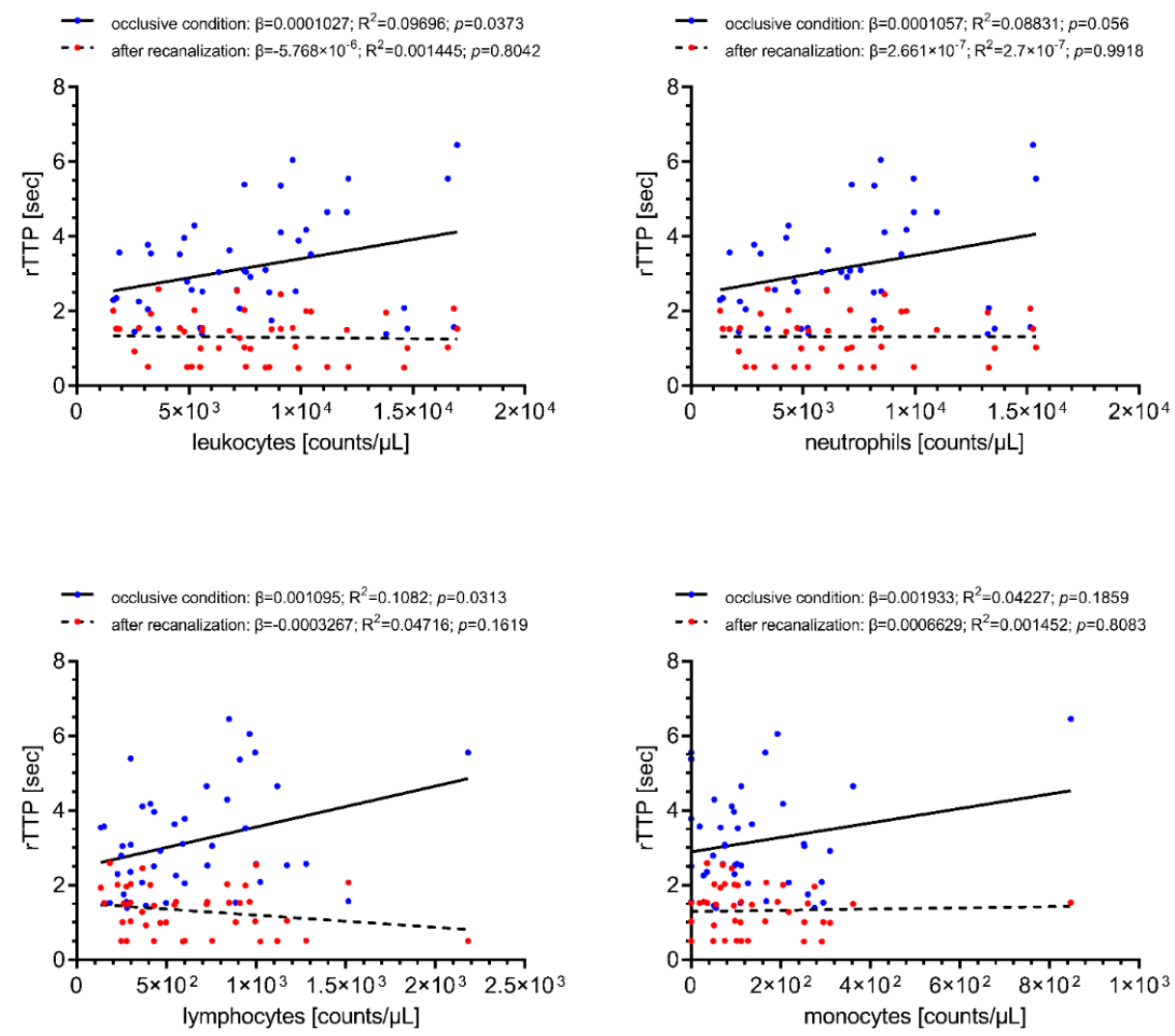

Figure 2. Simple linear regression of local immune cell counts with collateral transit time during occlusive conditions (blue) and antegrade blood flow (red) following recanalization (rTTP). Increased cerebral-ischemic total leukocyte and lymphocyte counts are associated with longer collateral transit time. Total leukocyte counts, $n=57$; differential leukocyte counts, $n=52$. rTTP, relative time to peak opacification; $\mathrm{R}^{2}$, coefficient of determination; $\beta$, regression coefficient.

\subsection{Association of Cerebral Immune Cell Recruitment with Infarct Extent}

Associations of cerebral-ischemic immune cell recruitment with baseline and followup infarct extent as assessed by ASPECTS are shown in Figure 3. Local cerebral-ischemic total leukocyte counts were correlated with reduced baseline $\left(\beta=-0.0001149, R^{2}=0.08382\right.$, $p=0.032)$ and follow-up $\left(\beta=-0.0001299, \mathrm{R}^{2}=0.07097, p=0.0472\right)$ ASPECTS, translating into a decrease of 1 baseline ASPECTS point for every increase of 8211 total leukocyte counts $/ \mu \mathrm{L}$. Local neutrophil counts were also correlated with infarct extent prior to recanalization $\left(\beta=-0.0001218, R^{2}=0.08878, p=0.0356\right)$. None of the other differential leukocyte counts was associated with infarct extent on baseline or follow-up imaging.

\subsection{Immune Cell Profiles in AIS Patients with Cervical ICA Tandem Lesions}

We additionally and independently analyzed immune cell counts in the subgroup of patients $(n=23)$ with tandem lesions at the cervical ICA level (i.e., stenosis $>50 \%$, percutaneous transluminal angioplasty, stenting, and ipsilateral dissection). These data are given in Figure 4. Intraindividual analyses of these patients (i.e., immune cell counts from ICA lesion level vs. cerebral-ischemic immune cell counts) revealed a shift towards higher total and differential leukocyte counts at the ICA lesion level, which was statistically significant for monocytes (+29.3\%, ICA lesion: $128 / \mu \mathrm{L}, 95 \%$ CI 74 to 182 vs. I: $99 / \mu \mathrm{L}$, $95 \%$ CI 60 to $138, p=0.0426)$. 

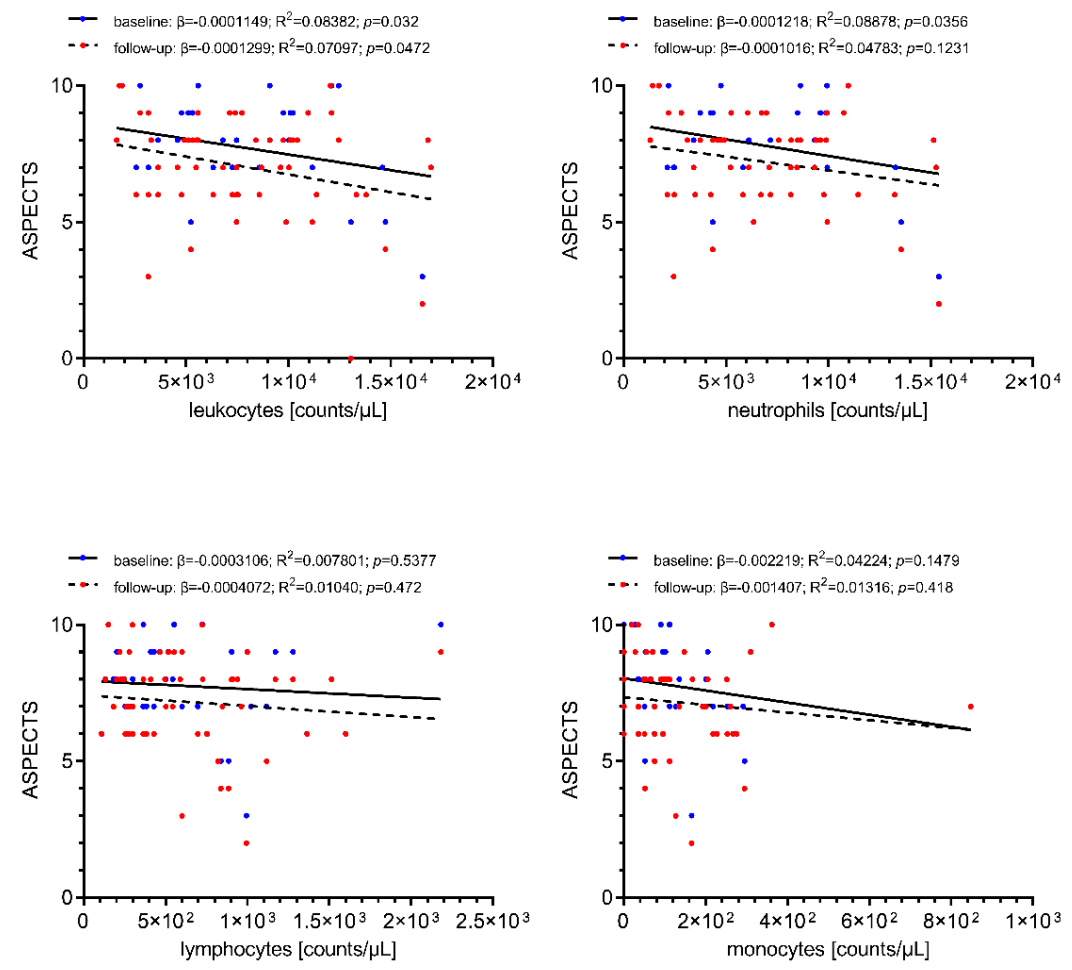

Figure 3. Simple linear regression of local immune cell counts with pre-interventional infarct extent at stroke center presentation (blue) and early follow-up infarct extent (ASPECTS) at 24-48 h following recanalization (red). Increased cerebral-ischemic total leukocyte and neutrophil counts are correlated with reduced baseline and follow-up ASPECTS (statistically significant only for total leukocyte counts). Total leukocyte counts, $n=58$; differential leukocyte counts, $n=54$. ASPECTS, Alberta Stroke Program Early CT score; $\mathrm{R}^{2}$, coefficient of determination; $\beta$, regression coefficient.
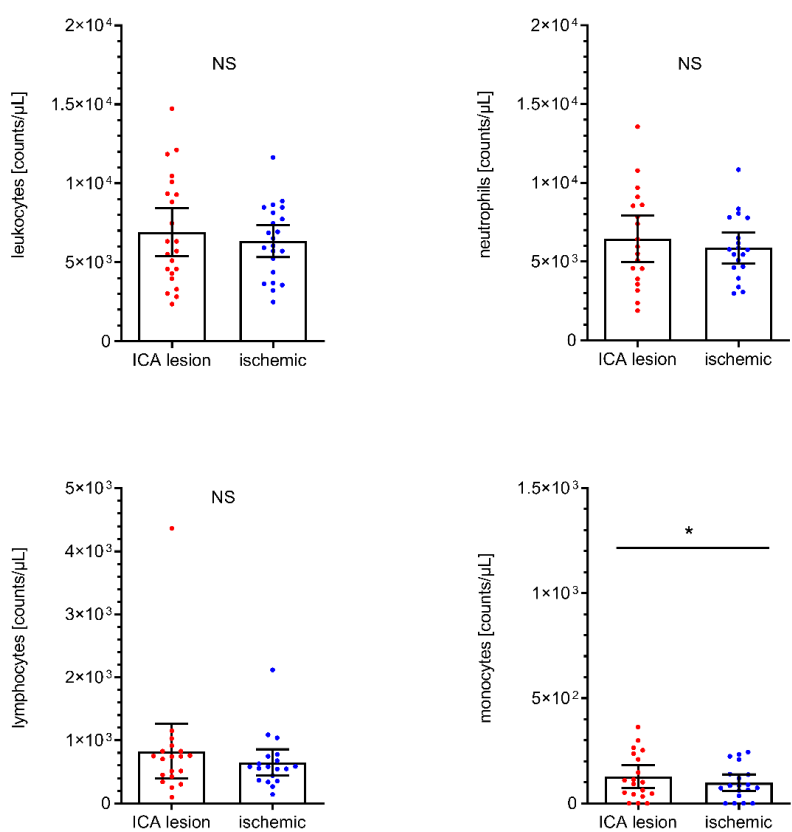

Figure 4. Analyses of total leukocyte $(n=22)$, neutrophil $(n=19)$, lymphocyte $(n=19)$ and monocyte $(n=19)$ cell counts in AIS patients with tandem lesion at ipsilateral cervical ICA level (including stenosis $>50 \%$, transluminal angioplasty/stenting, and ICA dissection). Scatter dot plot with mean and $95 \%$ confidence interval (CI). Total leukocyte cell, neutrophil, and monocyte counts were tested by paired T-test. Lymphocyte counts were tested by the Wilcoxon rank-sum test. ${ }^{*} p<0.05$. NS, not significant. ICA, internal carotid artery. 


\section{Discussion}

AIS elicits a strong innate inflammatory response, which emerges from the vascular compartment and which is directed at the ischemic brain region [22,23]. As the dominant constituents of this response, (1) local immune cell infiltration, (2) platelet activation, and (3) the interaction between infiltrating immune cells and activated platelets could be identified predominantly in experimental stroke models [5,11,24]. It would represent significant progress for translational stroke research to observe parts of these responses reliably and reproducibly in a more direct manner within the human cerebral vasculature $[15,16]$. In the pursuit of this aim, few groups, including ours, were able to establish a novel method by which blood samples can be obtained with a microcatheter from within the ischemic territory during MT for acute LVO stroke [17-19]. With this method, immune cells can be harvested directly from the occluded cerebral vascular compartment immediately before it is reopened by stent-embolus-retrieval. In other words, such local observation was not disturbed due to non-ischemic forward (antegrade) blood flow because sample aspiration was performed under strictly occlusive conditions [17]. As opposed to myocardial infarction, this unique opportunity has emerged in LVO stroke because the culprit lesion, in the vast majority of cases, is represented by an embolus, which can be penetrated with a microcatheter without concomitant vessel recanalization $[17,25,26]$.

In previous work, we established an extensive prospective protocol defining the flow of consecutive patients to homogenize the target population [17]. In addition, clinicalradiological, angiographic-interventional, and sampling-related variables were highly standardized. Importantly, protocol composition was guided by closely considering current concepts of the stroke-related immune response and vascular inflammation [5,11,15,27-29]. A central aspect in this regard was the control standard to which samples from the occluded cerebral vascular compartment were compared. To achieve a local (cerebral arterial), interindividual control standard remains elusive because invasive intervention and blood aspiration from corresponding cerebrovascular sites is not possible in non-stroke control subjects for ethical reasons [30]. To alternatively achieve a systemic (peripheral venous) inter-individual control standard for immune cell counts also remains elusive because such independent controls are inherently not comparable to acute inflammatory responses in the arterial compartment of the brain vasculature [31]. In addition, there is a substantial variation of systemic immune cell counts even between healthy individuals [32]. Sample sizes of smaller dimensions due to a complex study protocol, including many variables of prospective patient flow, sample aspiration, sample processing, and laboratory analysis, cannot overcome such a degree of variation [17,32]. For these reasons, one must argue for an intra-individual control standard of comparison. To be able to test and re-test the specific hypothesis of cerebral immune cell infiltration in the human system, intraindividual conditions where vascular inflammation and cell activation at a given systemic control level could confound local observation at the cerebrovascular target level need to be excluded by protocol.

There is evidence that atherosclerotic plaques within ICA stenoses upon inflammationdriven rupture per se activate platelets and the coagulation system, leading to cervical thrombus formation as a major cause of embolic ICA and MCA occlusions [33]. In line with these findings, elevated levels of neutrophil extracellular traps (NETs), activated platelets, and markers of platelet-derived microparticles have been reported at the site of cervical ICA occlusions in AIS patients [27]. To directly address this issue, we additionally analyzed immune cell counts in local cerebral-ischemic and carotid artery samples in an independent cohort of patients with ICA stenosis or dissection who were excluded from the primary cohort a priori. In this group of AIS patients, analyses in fact revealed numerically increased levels of immune cells at the site of cervical ICA tandem lesions. These data lend further empirical support to exclude any vascular lesion site as control for cerebral observation during AIS from a priori since they represent a confounding source of vascular inflammation themselves. In addition, several other protocol variables probably are also critical to becoming able to observe immune cell infiltration into the occluded cerebral 
vascular compartment. For example, in those LVO lesions where occlusion is not total but sub-occlusive (corresponding to mTICI 1), minimal residual forward (antegrade) blood flow passes into the ischemic compartment and may disturb local observation by contamination.

Thus far, we were able to prospectively and consecutively observe the two largest independent cohorts ( $n=318$ in this study, $n=183$ previously reported [17]) in which immune cells of AIS patients ( $n=58$ in this study, $n=40$ previously reported [17]) could be harvested from the occluded cerebral vascular compartment and analyzed with a high degree of standardization [34]. As a main finding of the current study, local immune cell infiltration into the occluded cerebral vascular compartment could be reproduced with very similar qualitative and quantitative observations in comparison to previous observations [17]. Specifically, it was replicated that neutrophils represent the dominant infiltrating immune cell population. In addition, the quantitative estimate of local vs. systemic neutrophil count was reproduced with considerable accuracy: Current replication of $+12.1 \%(p=0.0022)$ vs. previous finding of $+13.1 \%(p=0.0018)$ [17]. Since the literature suggests women have higher numbers of total leukocytes and neutrophil counts and have higher rates of overall complications, in-hospital deaths, and worse functional following AIS, our main finding of cerebral immune cell recruitment during AIS was additionally tested for sex differences in local immune cell counts which, however, could not be shown $[35,36]$.

We are led to believe that the human observation of local neutrophil infiltration into the occluded cerebral vascular compartment represents a true and consistent finding and no scientific or statistical error $[15,16]$. This finding is also highly plausible from a biological perspective within the experimental framework of ischemia-related inflammation: neutrophils are known to be among the first immune cells to invade the ischemic brain [28]. Furthermore, replication was possible in a second and independent cohort using identical a priori defined criteria of inclusion/exclusion without missing or selecting observations. The interpretation of our results may be further extended. Although we cannot directly prove the route and time course of neutrophil traffic, we may safely assume that, during complete LVO, vascular routes through leptomeningeal collateral (vascular) channels represent the major route of neutrophil infiltration at least during the very early phase after onset of cerebral ischemia [37]. This notion is fuelled by the experimental facts that neutrophils do not locally reside within but enter the brain only after pathological stimuli such as, e.g., ischemic stroke [28]. Further supporting this interpretation, regression analysis revealed the association of cerebral immune cell recruitment during occlusive conditions with dynamic (retrograde) blood flow (rTTP) and structural infarct extent (ASPECTS). These measures suggest that patients affected by slow (poor) collateral flow as well as those with more extensive structural damage show locally higher total leukocyte and particularly neutrophil density. Observations of this kind might reflect downstream effects of microvascular plugging and/or elevation of blood viscosity, which in turn could reduce retrograde collateral flow by increasing vascular resistance $[9,38]$.

In conclusion, the observation of neutrophil-dominant immune cell recruitment into the occluded cerebral vasculature of AIS patients could be prospectively and accurately reproduced in a large cohort by applying a highly standardized protocol as proposed by our group. Since we repeatedly detected this finding in a short-lived immune cell subpopulation, we recommend using this protocol for quality assurance of endovascular sampling, sample processing, and sample preparation. Future therapeutic breakthroughs of stroke immune modulation may depend on these novel methods of harvesting human immune cells directly, locally, and acutely during a stroke. In this manner, in-depth human immune cell phenotyping is becoming possible for reverse translation.

\section{Materials and Methods}

\subsection{Study Design}

This investigation was designed as a prospective cross-sectional study of consecutive ischemic stroke patients who underwent emergency MT due to acute symptomatic occlusion of the distal ICA, MCA M1, and proximal M2 segment between July 2019 to November 
2020. Briefly, we analyzed cerebral arterial blood obtained by microcatheter aspiration from within the ischemic cerebral vasculature, as previously reported by our group $[17,20,21]$. Ethical approval was obtained from the ethics committee of the University of Würzburg (\#135/17). All patients or their legal representatives provided written informed consent.

\subsection{Inclusion Criteria}

Patient inclusion criteria for the prospective assessment of local cerebral immune cell infiltration during AIS were defined as follows: (1) acute ischemic stroke (AIS) with severe neurological baseline deficit qualifying for mechanical thrombectomy (MT) according to current guidelines [3] and (2) periprocedural (invasive angiographic) confirmation of LVO of the following sites: distal ICA (ICA-T), MCA M1 segment or proximal M2 segment, respectively. Patients were excluded for the following reasons: (1) proven bilateral or multifocal LVO other than defined; (2) angiographically proven residual or restored antegrade blood flow [29]; (3) any deviation from the interventional, sampling, and preprocessing protocol; (4) LVO in conjunction with either $\geq 50 \%$ cervical ICA stenosis or ICA dissection; and (5) intraprocedural percutaneous transluminal angioplasty (PTA) or stent implantation [27].

Patient inclusion criteria for the prospective assessment of immune cell profiles in the subgroup of AIS patients with cervical ICA tandem lesions were defined as follows: (1) AIS with severe neurological baseline deficit qualifying for MT according to current guidelines [3]; (2) invasive angiographic confirmation of LVO of the following sites: distal ICA (ICA-T), MCA M1 segment or proximal M2 segment; and (3) LVO in conjunction with either $\geq 50 \%$ cervical ICA stenosis or ICA dissection; and (4) intraprocedural percutaneous transluminal angioplasty (PTA) or stent implantation [27]. Patients were excluded for the following reasons: (1) proven bilateral or multifocal LVO other than defined; (2) angiographically proven residual or restored antegrade blood flow [29]; (3) any deviation from the interventional, sampling, and pre-processing protocol.

\subsection{Interventional and Sampling Protocol}

Indication for MT by stent-embolus retrieval was given by acute symptomatic LVO [3]. Endovascular access for mechanical thrombectomy was obtained by a transfemoral approach using the modified Seldinger technique. Recanalization of the predefined target lesions was preceded by microcatheter navigation (Neuroslider 27 or 21; Acandis, Pforzheim, Germany) into the mid-insular middle cerebral artery M2 segment. The choice of a distinct microcatheter was at the discretion of the operator for reasons of variable vascular anatomy. After microcatheter positioning and discarding the respective microcatheter-specific dead space volume, a sample of $1 \mathrm{~mL}$ of local cerebral blood (ischemic) was drawn with $3 \mathrm{~mL}$ luer-lock syringe (Omnifix, B.Braun, Melsungen, Germany) if possible [17]. Samples obtained from the ipsilateral ICA were used as controls (systemic) and drawn analogously after clot removal and / or termination of the thrombectomy procedure [20,21]. All samples were immediately transferred into citrate-phosphate-dextrose-adenine (CPDA-1) monovettes (S-Monovette; Sarstedt, Nümbrecht, Germany) for anticoagulation.

\subsection{Radiological Parameters}

Pre-interventional infarct extent at stroke center presentation and early follow-up infarct extent at $24-48 \mathrm{~h}$ following recanalization were measured through the Alberta Stroke Program Early CT Score (ASPECTS; CT: Somatom Definition AS; Siemens Healthineers, Erlangen, Germany) [39]. Post-processing algorithms (syngo iFLOW software; Siemens Healthineers, Erlangen, Germany) were applied for parametric color coding of digital subtraction angiography (DSA) series and for the quantification of collateralization during occlusive conditions. The retrograde collateral flow was assessed by means of collateral transit time as defined by relative time to peak opacification (rTTP). rTTP was determined by using a region-of-interest (ROI) analysis, where predetermined ROI locations reflected the site of local cerebral sampling and the mid-insular target region receiving retrograde 
blood supply under occlusion conditions. rTTP was calculated by subtracting the time to peak opacification (TTP) of ROI 1 (circular, placed at the lacerum segment of the internal carotid artery, measuring $10.80 \mathrm{~mm}^{2}$ ) from the TTP of ROI 2 (circular, placed centered at the mid-insular sampling location, measuring $309.60 \mathrm{~mm}^{2}$ ) [40]. The angiographic degree of final recanalization following MT was graded by the modified treatment in cerebral infarction scale (mTICI) [41]. Antegrade macrovascular blood flow was assessed by means of rTTP at the site of local cerebral sampling following final recanalization.

\subsection{Laboratory Analysis}

A time window $\leq 12 \mathrm{~h}$ after completion of sampling was allowed for sample processing. All laboratory analyses were performed by trained personnel unaware of clinical data. The CPDA-1-anticoagulated blood was used for cell counting and the preparation of blood smears. After red blood cell lysis and white blood cell (WBC) staining with Tuerk solution (Merck, Darmstadt, Germany), total WBC counts were determined by using a Fuchs Rosenthal hemocytometer. Thin blood smears were prepared from $5 \mu \mathrm{L}$ of whole blood (glass slides: R. Langenbrinck, Emmendingen, Germany). Blood smears were stained by standard Pappenheim stain (Merck). Cell counts of neutrophils, lymphocytes, and monocytes were calculated by multiplying the percentages in Pappenheim-stained blood smears with the respective WBC counts.

\subsection{Prospective Patient Flow}

The total eligible cohort comprised $n=318$ patients who presented with suspected LVO between July 2019 and November 2020. A flowchart of patient inclusion is provided in Figure 5.

Of these $n=318$ patients, $n=43$ were excluded for vertebrobasilar occlusion sites, and $n=14$ patients for bilateral or multifocal occlusions other than the ipsilateral ICA or MCA. Of the remaining $n=261$ patients, $n=53$ patients failed to meet study inclusion criteria either because of angiographically proven sub-occlusion with residual antegrade blood flow or complete recanalization. Intraprocedural microcatheter aspiration of local cerebral-ischemic blood samples was attempted in $n=208$ patients. According to the study protocol, precise occlusion locations were angiographically proven and limited to the following sites of anterior circulation LVO: ICA-T, MCA M1, and proximal M2 segment. Local microcatheter aspiration was attempted in all $n=208$ patients but remained empty (sicca) in $n=83 / 208$ patients. It succeeded in $n=125 / 208$ patients $(60 \%)$. Of these $n=125$ patients, $n=19$ patients were excluded for protocol violations of the sampling technique or sampling order (e.g., inadvertent reversal of sampling order local vs. systemic, non-insular target location, omission of second/control sample). $\mathrm{N}=23$ patients were excluded because of the relevant tandem lesion at cervical level (stenosis $>50 \%$ ) with or without subsequent percutaneous transluminal angioplasty (PTA) and/or stenting, also including cases with primary or secondary dissection of ipsilateral cervical ICA [42,43]. These cervical tandem lesions were considered as potentially powerful confounders from a priori since local vascular inflammation should be expected at these cervical sites [27]. $\mathrm{N}=25$ patients could not be included for reasons of poor sample quality or of deviation from the sample pre-processing protocol (insufficient sample volume or time delay $>12 \mathrm{~h}$ between sampling and sample analysis). At the end of consecutive patient flow, $n=58$ patients met all a priori defined clinical-radiological, sampling, interventional, and laboratory criteria of study inclusion.

\subsection{Statistical Analysis}

Statistical analyses were performed using GraphPad Prism (GraphPad Prism 9.0.0, GraphPad Software, San Diego, CA, USA) and MedCalc 19.6 (MedCalc Software, Ostend, Belgium). The normal distribution of datasets was tested using the D'Agostino-Pearsons test. Data are given as mean with $95 \%$ confidence interval (CI), as mean \pm standard deviation (SD), as median with interquartile range (IQR), or absolute and relative frequency distribution unless otherwise specified. The paired t-test or Wilcoxon Rank-Sum test was 
used to compare cell counts of related samples. The unpaired t-test or Mann-Whitney U test was used to compare cell counts of unrelated samples. Simple linear regression was performed to identify associations between cerebral-ischemic cell counts, hemodynamicfunctional, and radiological-structural parameters. All $p$ values reported were 2-sided with $p<0.05$ being considered statistically significant.

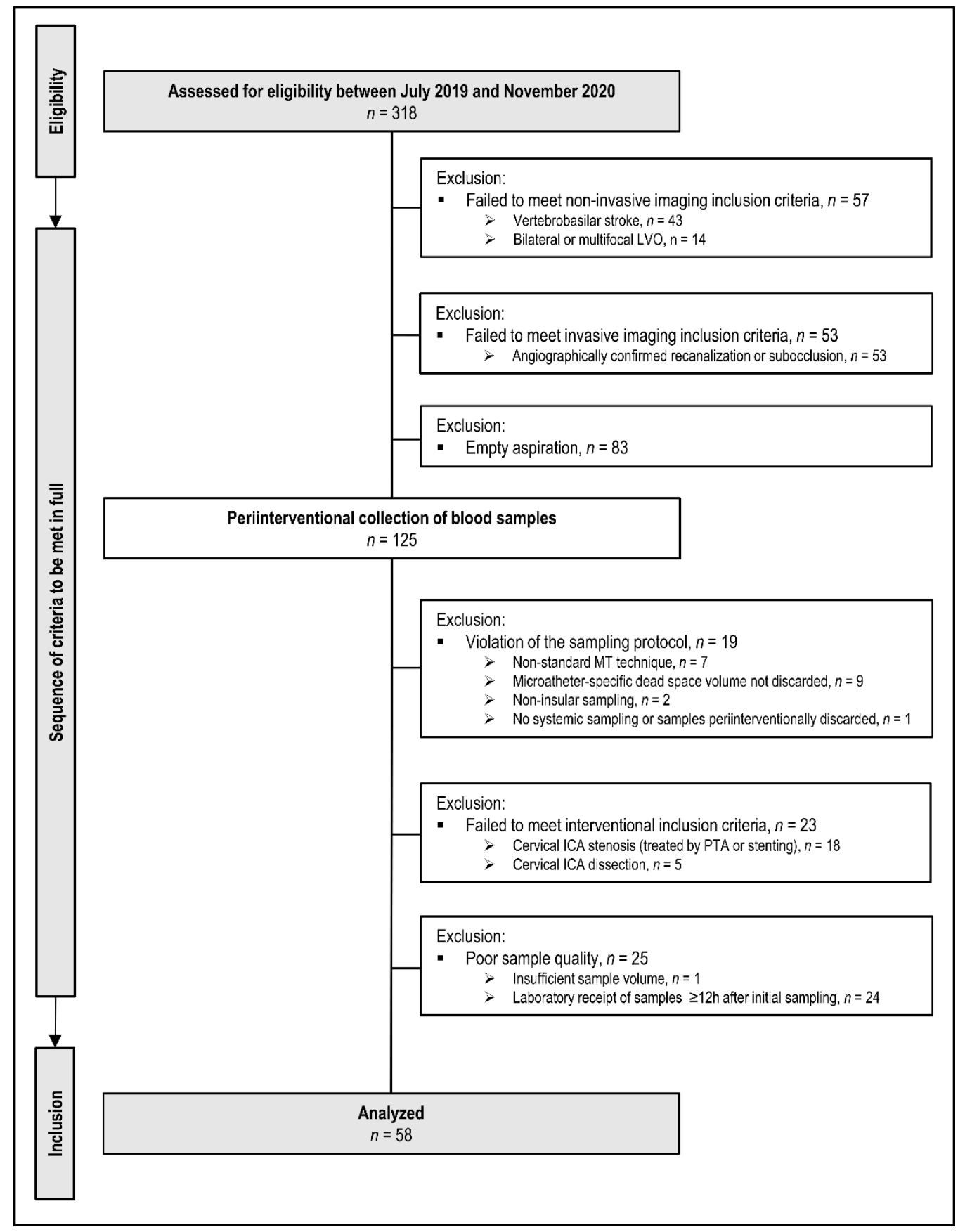

Figure 5. Flow chart of patient inclusion and exclusion according to the prospective protocol (multiple exclusion criteria possible). Non-standard abbreviations: ICA, internal carotid artery; LVO, large-vessel-occlusion; MT, mechanical thrombectomy; PTA, percutaneous transluminal angioplasty.

\subsection{Manuscript Preparation}

The manuscript was prepared according to the STROBE (Strengthening the Reporting of Observational Studies in Epidemiology) statement for observational studies [44]. 
Author Contributions: Conceptualization, M.P., G.S. and A.M.K.; methodology, M.S. and M.K.S.; validation, M.S. and A.M.K.; formal analysis, M.S. and A.M.K.; investigation, M.S.; resources, M.P. and M.K.S.; data curation, A.G.M., J.F., F.W., M.L.V., F.E., H.N., G.S.; writing-original draft preparation, M.S.; writing-review and editing, M.P., G.S. and A.M.K.; visualization, M.S. and A.M.K.; supervision, A.M.K.; project administration, M.P.; funding acquisition, M.P., M.K.S., G.S. and A.M.K. All authors have read and agreed to the published version of the manuscript.

Funding: Deutsche Forschungsgemeinschaft (project number 374031971—CRC/TR 240, project number 413657723-UNION CVD).

Institutional Review Board Statement: The study was conducted in accordance with the Declaration of Helsinki and approved by the Ethics Committee of the Medical Faculty of the University of Würzburg, Germany (approval nr. 135/17; 03/16/2018, 06/17/2020).

Informed Consent Statement: Informed consent was obtained from all individuals included in the study or from their legal representatives.

Data Availability Statement: The datasets generated during and/or analyzed during the current study are available from the corresponding author on reasonable request.

Acknowledgments: We thank Yanyan Xiong, Julian Kunz and Thomas Günthner-Lengsfeld for their support in data acquisition and patient handling. The authors thank Saskia Moritz, Gabriele Köllner, and Susi Hellmig for excellent technical support.

Conflicts of Interest: The authors declare no conflict of interests.

\section{References}

1. Marrugat, J.; Arboix, A.; García-Eroles, L.; Salas, T.; Vila, J.; Castell, C.; Tresserras, R.; Elosua, R. The estimated incidence and case fatality rate of ischemic and hemorrhagic cerebrovascular disease in 2002 in Catalonia. Rev. Esp. Cardiol. 2007, 60, 573-580. [CrossRef]

2. Feigin, V.L.; Lawes, C.M.M.; Bennett, D.A.; Barker-Collo, S.L.; Parag, V. Worldwide stroke incidence and early case fatality reported in 56 population-based studies: A systematic review. Lancet Neurol. 2009, 8, 355-369. [CrossRef]

3. Powers, W.J.; Rabinstein, A.A.; Ackerson, T.; Adeoye, O.M.; Bambakidis, N.C.; Becker, K.; Biller, J.; Brown, M.; Demaerschalk, B.M.; Hoh, B.; et al. Guidelines for the Early Management of Patients With Acute Ischemic Stroke: 2019 Update to the 2018 Guidelines for the Early Management of Acute Ischemic Stroke: A Guideline for Healthcare Professionals From the American Heart Association/American Stroke. Stroke 2019, 50, E344-E418. [CrossRef] [PubMed]

4. GBD 2015 Neurological Disorders Collaborator Group. Global, regional, and national burden of neurological disorders during 1990-2015: A systematic analysis for the Global Burden of Disease Study 2015. Lancet Neurol. 2017, 16, 877-897. [CrossRef]

5. Iadecola, C.; Buckwalter, M.S.; Anrather, J. Immune responses to stroke: Mechanisms, modulation, and therapeutic potential. J. Clin. Investig. 2020, 130, 2777-2788. [CrossRef] [PubMed]

6. Chamorro, Á.; Dirnagl, U.; Urra, X.; Planas, A.M. Neuroprotection in acute stroke: Targeting excitotoxicity, oxidative and nitrosative stress, and inflammation. Lancet Neurol. 2016, 15, 869-881. [CrossRef]

7. del Zoppo, G.J. Inflammation and the neurovascular unit in the setting of focal cerebral ischemia. Neuroscience 2009, 158, 972-982. [CrossRef]

8. Sirén, A.L.; Heldman, E.; Doron, D.; Lysko, P.G.; Yue, T.L.; Liu, Y.; Feuerstein, G.; Hallenbeck, J.M. Release of proinflammatory and prothrombotic mediators in the brain and peripheral circulation in spontaneously hypertensive and normotensive Wistar-Kyoto rats. Stroke 1992, 23, 1643-1650. [CrossRef]

9. del Zoppo, G.J.; Schmid-Schönbein, G.W.; Mori, E.; Copeland, B.R.; Chang, C.M. Polymorphonuclear leukocytes occlude capillaries following middle cerebral artery occlusion and reperfusion in baboons. Stroke 1991, 22, 1276-1283. [CrossRef]

10. Beuker, C.; Strecker, J.-K.; Rawal, R.; Schmidt-Pogoda, A.; Ruck, T.; Wiendl, H.; Klotz, L.; Schäbitz, W.-R.; Sommer, C.J.; Minnerup, H.; et al. Immune Cell Infiltration into the Brain After Ischemic Stroke in Humans Compared to Mice and Rats: A Systematic Review and Meta-Analysis. Transl. Stroke Res. 2021. [CrossRef]

11. Stoll, G.; Nieswandt, B. Thrombo-inflammation in acute ischaemic stroke-Implications for treatment. Nat. Rev. Neurol. 2019, 15, 473-481. [CrossRef]

12. Allan, S.M.; Tyrrell, P.J.; Rothwell, N.J. Interleukin-1 and neuronal injury. Nat. Rev. Immunol. 2005, 5, 629-640. [CrossRef]

13. Krams, M.; Lees, K.R.; Hacke, W.; Grieve, A.P.; Orgogozo, J.-M.; Ford, G.A. ASTIN Study Investigators Acute Stroke Therapy by Inhibition of Neutrophils (ASTIN): An adaptive dose-response study of UK-279,276 in acute ischemic stroke. Stroke 2003, 34, 2543-2548. [CrossRef] [PubMed]

14. Veltkamp, R.; Gill, D. Clinical Trials of Immunomodulation in Ischemic Stroke. Neurotherapeutics 2016, 13, 791-800. [CrossRef] [PubMed]

15. Dirnagl, U. Thomas Willis Lecture. Stroke 2016, 47, 2148-2153. [CrossRef] 
16. Begley, C.G.; Ioannidis, J.P.A. Reproducibility in science: Improving the standard for basic and preclinical research. Circ. Res. 2015, 116, 116-126. [CrossRef] [PubMed]

17. Kollikowski, A.M.; Schuhmann, M.K.; Nieswandt, B.; Müllges, W.; Stoll, G.; Pham, M. Local Leukocyte Invasion during Hyperacute Human Ischemic Stroke. Ann. Neurol. 2020, 87, 466-479. [CrossRef] [PubMed]

18. Fraser, J.F.; Collier, L.A.; Gorman, A.A.; Martha, S.R.; Salmeron, K.E.; Trout, A.L.; Edwards, D.N.; Davis, S.M.; Lukins, D.E.; Alhajeri, A.; et al. The Blood And Clot Thrombectomy Registry And Collaboration (BACTRAC) protocol: Novel method for evaluating human stroke. J. Neurointerv. Surg. 2019, 11, 265-270. [CrossRef] [PubMed]

19. Flores, A.; Sargento-Freitas, J.; Pagola, J.; Rodriguez-Luna, D.; Piñeiro, S.; Maisterra, O.; Rubiera, M.; Montaner, J.; Alvarez-Sabin, J.; Molina, C.; et al. Arterial blood gas analysis of samples directly obtained beyond cerebral arterial occlusion during endovascular procedures predicts clinical outcome. J. Neuroimaging 2013, 23, 180-184. [CrossRef]

20. Essig, F.; Kollikowski, A.M.; Müllges, W.; Stoll, G.; Haeusler, K.G.; Schuhmann, M.K.; Pham, M. Local Cerebral Recombinant Tissue Plasminogen Activator Concentrations During Acute Stroke. JAMA Neurol. 2021, 78, 615-617. [CrossRef]

21. Schuhmann, M.K.; Kollikowski, A.M.; März, A.G.; Bieber, M.; Pham, M.; Stoll, G. Danger-associated molecular patterns are locally released during occlusion in hyper-acute stroke. Brain Behav. Immun. Health 2021, 15, 100270. [CrossRef]

22. Buck, B.H.; Liebeskind, D.S.; Saver, J.L.; Bang, O.Y.; Yun, S.W.; Starkman, S.; Ali, L.K.; Kim, D.; Villablanca, J.P.; Salamon, N.; et al. Early neutrophilia is associated with volume of ischemic tissue in acute stroke. Stroke 2008, 39, 355-360. [CrossRef]

23. Iadecola, C.; Anrather, J. The immunology of stroke: From mechanisms to translation. Nat. Med. 2011, 17, 796-808. [CrossRef] [PubMed]

24. Schuhmann, M.K.; Bieber, M.; Franke, M.; Kollikowski, A.M.; Stegner, D.; Heinze, K.G.; Nieswandt, B.; Pham, M.; Stoll, G. Platelets and lymphocytes drive progressive penumbral tissue loss during middle cerebral artery occlusion in mice. J. Neuroinflamm. 2021, 18, 46. [CrossRef] [PubMed]

25. De Meyer, S.F.; Andersson, T.; Baxter, B.; Bendszus, M.; Brouwer, P.; Brinjikji, W.; Campbell, B.C.V.; Costalat, V.; Dávalos, A.; Demchuk, A.; et al. Analyses of thrombi in acute ischemic stroke: A consensus statement on current knowledge and future directions. Int. J. Stroke 2017, 12, 606-614. [CrossRef] [PubMed]

26. Maier, W.; Altwegg, L.A.; Corti, R.; Gay, S.; Hersberger, M.; Maly, F.E.; Sütsch, G.; Roffi, M.; Neidhart, M.; Eberli, F.R.; et al. Inflammatory markers at the site of ruptured plaque in acute myocardial infarction: Locally increased interleukin- 6 and serum amyloid A but decreased C-reactive protein. Circulation 2005, 111, 1355-1361. [CrossRef] [PubMed]

27. Zhou, P.; Li, T.; Jin, J.; Liu, Y.; Li, B.; Sun, Q.; Tian, J.; Zhao, H.; Liu, Z.; Ma, S.; et al. Interactions between neutrophil extracellular traps and activated platelets enhance procoagulant activity in acute stroke patients with ICA occlusion. EBioMedicine 2020, 53, 102671. [CrossRef]

28. Strecker, J.-K.; Schmidt, A.; Schäbitz, W.-R.; Minnerup, J. Neutrophil granulocytes in cerebral ischemia-Evolution from killers to key players. Neurochem. Int. 2017, 107, 117-126. [CrossRef]

29. Sommer, C.J. Ischemic stroke: Experimental models and reality. Acta Neuropathol. 2017, 133, 245-261. [CrossRef]

30. Emanuel, E.J.; Wendler, D.; Grady, C. What makes clinical research ethical? JAMA 2000, 283, 2701-2711. [CrossRef]

31. Denes, A.; Thornton, P.; Rothwell, N.J.; Allan, S.M. Inflammation and brain injury: Acute cerebral ischaemia, peripheral and central inflammation. Brain Behav. Immun. 2010, 24, 708-723. [CrossRef]

32. Ross, D.W.; Ayscue, L.H.; Watson, J.; Bentley, S.A. Stability of hematologic parameters in healthy subjects. Intraindividual versus interindividual variation. Am. J. Clin. Pathol. 1988, 90, 262-267. [CrossRef]

33. Stoll, G.; Bendszus, M. Inflammation and atherosclerosis: Novel insights into plaque formation and destabilization. Stroke 2006, 37, 1923-1932. [CrossRef] [PubMed]

34. Shaw, B.C.; Maglinger, G.B.; Ujas, T.; Rupareliya, C.; Fraser, J.F.; Grupke, S.; Kesler, M.; Gelderblom, M.; Pennypacker, K.R.; Turchan-Cholewo, J.; et al. Isolation and identification of leukocyte populations in intracranial blood collected during mechanical thrombectomy. J. Cereb. Blood Flow Metab. 2021, 271678X211028496. [CrossRef] [PubMed]

35. Arboix, A.; Cartanyà, A.; Lowak, M.; García-Eroles, L.; Parra, O.; Oliveres, M.; Massons, J. Gender differences and woman-specific trends in acute stroke: Results from a hospital-based registry (1986-2009). Clin. Neurol. Neurosurg. 2014, 127, 19-24. [CrossRef] [PubMed]

36. Bain, B.J. Ethnic and sex differences in the total and differential white cell count and platelet count. J. Clin. Pathol. 1996, 49, 664-666. [CrossRef] [PubMed]

37. Vander Eecken, H.M.; Adams, R.D. The Anatomy and Functional Significance of the Meningeal Arterial Anastomoses of the Human Brain. J. Neuropathol. Exp. Neurol. 1953, 12, 132-157. [CrossRef]

38. Pham, M.; Bendszus, M. Facing Time in Ischemic Stroke: An Alternative Hypothesis for Collateral Failure. Clin. Neuroradiol. 2016, 26, 141-151. [CrossRef]

39. Kollikowski, A.M.; Cattus, F.; Haag, J.; Feick, J.; März, A.G.; Weidner, F.; Schuhmann, M.K.; Müllges, W.; Stoll, G.; Pham, M.; et al. Progression of cerebral infarction before and after thrombectomy is modified by prehospital pathways. J. Neurointerv. Surg. 2021. [CrossRef]

40. Gölitz, P.; Muehlen, I.; Gerner, S.T.; Knossalla, F.; Doerfler, A. Ultraearly assessed reperfusion status after middle cerebral artery recanalization predicting clinical outcome. Acta Neurol. Scand. 2018, 137, 609-617. [CrossRef] 
41. Zaidat, O.O.; Yoo, A.J.; Khatri, P.; Tomsick, T.A.; von Kummer, R.; Saver, J.L.; Marks, M.P.; Prabhakaran, S.; Kallmes, D.F.; Fitzsimmons, B.-F.M.; et al. Recommendations on angiographic revascularization grading standards for acute ischemic stroke: A consensus statement. Stroke 2013, 44, 2650-2663. [CrossRef] [PubMed]

42. Chen, W.-T.; Chang, F.-C.; Huang, H.-C.; Tsai, J.-Y.; Chung, C.-P. Total and differential leukocyte counts in ischemic stroke caused by vertebrobasilar artery dissection. J. Neurol. Sci. 2019, 404, 101-105. [CrossRef] [PubMed]

43. Welt, F.G.P.; Rogers, C. Inflammation and restenosis in the stent era. Arterioscler. Thromb. Vasc. Biol. 2002, 22, 1769-1776. [CrossRef]

44. von Elm, E.; Altman, D.G.; Egger, M.; Pocock, S.J.; Gøtzsche, P.C.; Vandenbroucke, J.P. STROBE Initiative The Strengthening the Reporting of Observational Studies in Epidemiology (STROBE) Statement: Guidelines for reporting observational studies. Int. J. Surg. 2014, 12, 1495-1499. [CrossRef] [PubMed] 